\title{
Specialised care for early psychosis may reduce number of readmissions
}

Craig TK, Garety P, Power P, et al. The Lambeth Early Onset (LEO) Team: randomised controlled trial of the effectiveness of specialised care for early psychosis. BMJ 2004;329:1067-70.

What is the effect of a specialised care team for early psychosis on relapse and readmissions?

\section{METHODS}

$\square$

Design: Randomised controlled trial

$\lambda$

Allocation: Concealed

Blinding: Single blinded (assessors blinded)

Follow up period: 18 months

Setting: Mental health service in Lambeth, London, UK; recruitment January 2000 to October 2001

Patients: 144 adults (16-40 years old) with non-affective psychosis (ICD-10 diagnosis of schizophrenia, schizotypal, or delusional disorder) presenting to psychiatric services for the first or second time, with no previous routine community mental health treatment. Excluded were asylum seekers, and people with organic psychosis or a primary substance addiction.

$\mathbf{R}_{\mathbf{X}}$

Intervention: Specialised assertive outreach care by a multidisciplinary community mental health team, who provided extended access to care (weekends and holidays), and individually tailored evidence-based treatment including low dose atypical antipsychotic drugs, cognitive behavioural therapy, family counselling, and vocational strategies as necessary; standard care by community mental health teams who were encouraged to follow existing treatment guidelines but did not receive extra training in early psychosis management.

Outcomes: Primary outcomes: rate of relapse; readmission to hospital. Secondary outcome: recovery (either full or partial) from the index episode.

$\square \square$ Patient follow up: $91 \%$ at 18 months.

\section{MAIN RESULTS}

The chance of recovery from the index episode was similar in specialised and standard care groups (61/69 (90\%) with specialised care $v 61 / 67$ (91\%) with standard care; adjusted OR 1.76, 95\% CI 0.51 to 6.09$)$. Specialised care reduced risk of relapse, but this reduction was not significant after adjustment for confounding factors (18/61 (30\%) with specialised care $v 29 / 61$ (48\%) with standard care; OR adjusted for gender, previous psychotic episodes, and ethnic group: $0.55,95 \%$ CI 0.24 to 1.26 ). Specialised care did not reduce proportion of people needing readmission, but significantly reduced mean number of readmissions compared with standard care (AR 33\% with specialised care $v 51 \%$ with standard care, adjusted OR $0.53,95 \%$ CI 0.26 to 1.12 ; mean number of admissions 0.4 with specialised care $v$ 0.8 with standard care; OR $0.36,95 \%$ CI 0.04 to 0.66 ). For correspondence: Professor Tom K J Craig, Institute of Psychiatry, De Crespigny Park, London SE5 8AF, UK

Sources of funding: Directorate of Health and Social Care London Research and Development Organisation and Management Programme.

\section{CONCLUSIONS}

There is some preliminary evidence that providing specialised care for people with early psychosis may reduce the number of readmissions.

\section{NOTES}

The authors reported that the study was underpowered, and that correction for multiple statistical comparisons would render the readmission results non-significant.

\section{Commentary}

chizophrenia has traditionally been seen as a chronic psychotic condition in which poor outcome was inevitable. There has been a surge of enthusiasm about the possibility that earlier identification and treatment for schizophrenia can lead to dramatically improved outcomes. The UK, in particular, has invested heavily in the development of specialised assertive treatment teams for early intervention in psychotic illnesses. Two key aspects of early intervention have captured much of the attention in the field: (1) providing comprehensive treatment specialised to meet the needs of people in the early phases of illness; and (2) intervening as early as possible after the onset of psychotic symptoms so as to limit the duration of untreated psychosis (DUP), a putative mediator of outcome.

This study attempts to address the first issue by randomising participants at the time of first presentation to either a recently established assertive outreach team for early psychosis or to standard care with a local community mental health team. As randomisation occurred after referral, this study does not address the question of whether specialised early intervention teams will lead to shorter DUPs.

The results do highlight the notion that outcomes are not fixed features of schizophrenia per se, but rather reflect the impact of interventions and patients' adherence to them. What we do not know is which interventions are critical in accounting for these differences in outcome. Unfortunately, neither of the interventions was well characterised in the published report. Specialised care was based on the principles of assertive outreach with extended hours of service, together with low dose atypical antipsychotics, cognitive-behavioral therapy, family counselling, and vocational support. The components of standard care were not specified. Although it is tempting to believe that it is the comprehensive approach embodied in 'specialised care' which accounts for the differences found, there is no evidence presented to support this conclusion. For example, we know that the specialised care team was prescribing atypical antipsychotics; while there is little reason to believe that these medications are more efficacious than typical antipsychotics, there is good reason to believe that first episode patients are less likely to discontinue them. ${ }^{12}$ This in itself could contribute to the differences reported. In the end, this study leaves the reader uncertain of what it is about specialised care that does make a difference. Further studies will be required to identify the critical interventions that mediate the improvements in rates of relapse, readmission, and recovery found in this study.

Robert B Zipursky, MD FRCP(C) University of Toronto, Toronto, Canada

1 Lieberman JA, Tollefson G, Tohen MD, et al. Comparative efficacy and safety of atypical and conventional antipsychotic drugs in first-episode psychosis: a randomized double-blind trial of olanzapine vs haloperidol. Am J Psychiatry 2003;160:1396-404.

2 Emsley RA. Risperidone in the treatment of first-episode psychotic patients: a double-blind multicenter study. Schizophr Bull 1999:25:721-9. 